content of AngII. Apoptosis percentage of myocardial cell of activating blood circulation group was lower than that of benefiting vital energy group.

Conclusions Recipe for activating blood circulation and benefiting vital energy can inhibit left ventricular remodelling and myocardial apoptosis, and delay development of heart failure. The effect of activating blood circulation group 8 weeks is the best in all group.

\section{GW23-e1771 COMPARISON OF RECIPE FOR ACTIVATING BLOOD CIRCULATION AND BENEFITING VITAL ENERGY ON INHIBITING LEFT VENTRICULAR REMODELLING AND APOPTOSIS IN RATS WITH HEART FAILURE}

doi:10.1136/heartjnl-2012-302920a.195
Objectives To compare the effective characteristic of recipe for activating blood circulation and benefiting vital energy on inhibiting left ventricular remodelling and apoptosis in rats with heart failure. Methods Left coronary artery occlusion was conducted to establish rat model of heart failure after myocardial infarction. The models were randomly divided into five groups, consisting of model, activating blood circulation, benefiting vital energy, activating blood circulation plus benefiting vital energy, western medicine (captopril). Sham operation group was negative control. The rats were treated on the second day after myocardial infarction and course of treatment was 4 weeks or 8 weeks respectively. Heart function was evaluated by impedance method. Patho-dyeing and image analysis were performed to determine perimeter and area of left ventricular cavity, myocardial nuclei number and collagen content per square area. Apoptosis percentage of myocardial cell was detected by TUNEL and content of AngII in cardiac muscle was measured by radio-immunity.

Results In comparison with the model group, function of left ventricular contraction was improved; area of left ventricular cavity was diminished; proliferation of collagen, content of AngII and apoptosis percentage of myocardial cell were reduced in every management group $p<0.05$ or $p<0.01$ ). The effect of activating blood circulation group 8 weeks was as similar as that of sham operation group in cardiac index (CI), area of left ventricular cavity and 